\title{
Review on In-Patient Hospital Stay
}

\author{
Garima Sharma \\ M.Tech Scholar \\ Department of Computer \\ Science \& Engineering \\ ABES, Engineering College, \\ Ghaziabad, India
}

\author{
Saurabh Kr. Srivastava \\ Sr. Assistant Professor \\ Department of Computer \\ Science \& Engineering \\ ABES, Engineering College, \\ Ghaziabad, India
}

\begin{abstract}
Forecasting In-Patient length of stay is a prime concern for patients in diagnosis of their diseases. It is also significant for hospitals to plan and manage their services for patients efficiently. Length of In-Patient stay prediction plays a crucial role in strategic decision making by healthcare administrators and also plan their resources. This paper presents a review of related algorithms and methods that are carried out by the researchers in past years. Most of the papers calculated outcomes in terms of MSE (mean square error) and AFER (average forecasting error rate) and had compared with different pre-existing methods. With the review of the literature identified that there is a still scope of some improvement required in healthcare domain using Genetic Algorithm approach.
\end{abstract}

\section{General Terms}

Time Series Forecasting: A time series is a collection of numerical data points which have taken over a specific period of time at regular interval of time in successive order whether weekly, monthly, quarterly, or yearly. For an example, Alabama university enrollment data set. The time series data used in various fields such as weather forecasting, signal processing, pattern recognition and big data analytics. The term time series analysis is the comprising method used for analyzing the time series data in order to extract meaningful information to solve a specific problem. Whereas, Time Series Forecasting refers to a model used to predict future values based on previously observed value. The forecasting analysis analyzes the trendiness of time series data.

\section{Keywords}

Fuzzy time series, soft computing, Linguistic data sets, Average length of stay (LOS), AFER and MSE.

\section{INTRODUCTION}

As look out in past, seemed that there is no proper efficient healthcare system for hospitals to efficiently plan and manage services for patients. Due to this people are facing many problems like: limited resources, incomplete knowledge about the time period need for disease treatment, disease diagnosis, and many more. When the people who are working in the private organizations was not able to know about the diagnosis of their diseases, due to incomplete information peoples are unable to schedule their work properly and suffer with great loss of work and money.

Now days, forecasting In-patient stay is becoming major concern for patients in disease diagnosis. So, that patients can efficiently plan and schedule their work properly where minimum work loss or no work loss. It is also important for patients to efficiently plan and manage their services. And also the hospital administrators can easily allocate resources to patients and provide them better healthcare treatment and facilities. Our aim is to forecast in-patient length of stay in terms of better healthcare. Forecasting plays significant role in patient healthcare plans and medical insurances plan. Past research work which was carried out in that reference, not able to provide an effective efficient model to measure patient length of stay. Some applications were built in that reference but those applications proved impractical to implement in hospitals in real life. So, it's become important to make a scientifically robust and efficient healthcare system which practical enough for implementation in healthcare systems.

\section{MOTIVATION OF WORK}

In past research work there is no significant scientific efficient model to forecast in-patient length of stay. So that it is beneficial for both patients and healthcare administrators to proposed practically possible healthcare model. The main aim of this paper is to present a literature survey in order to forecast efficient forecasting model in terms of better care. Where the hospital administrators can easily allocate resources to patients and provide the better healthcare treatment and facility. And patients plan their work accordingly and properly.

Forecasting prediction play a significant role inpatient healthcare plans and medical insurance plans. Some applications were built in that reference but those applications proved impractical to implement in hospitals in real life. So, it is important to develop scientifically robust, practically existent model for forecasting patient length of stay.

\section{REVIEW OF RELATED WORKS AND THEIR IMPLEMENTATIONS 3.1 Title: A Fuzzy Time Series and its Model}

Publisher: An article in Fuzzy Sets and Systems March (1993) 267-277 Elsevier Science Publisher B.V

Author: Q. Song, B.S Chissom

Theory: In this paper, the fuzzy time series and fuzzy relationship equation defined and also explained two classes of fuzzy time series that is time - variant and time - invariant. And also had mentioned the Mamdami's method used to compute fuzzy relationship using fuzzy data set.

\section{Critical Review:}

Methodology Used: The Mamdami's method used to compute fuzzy relationship through fuzzy time series data and then forecasted data used in forecasting procedure ${ }^{[1]}$ in fuzzy environment. For that, they had considered time - invariant fuzzy time series.

Data Used: historical data set

Paper Conclusion: Proposed paper explains fuzzy time series. 
In general, they differentiate traditional and fuzzy time series. And mentioned out one of the possible applications of fuzzy time series is a forecasting problem under fuzzy environments using historical data usually linguistic values. In order to apply time invariant fuzzy time series, they explored a simple procedure that was Mamdami's method.

Own Conclusion: In this Paper, explains fuzzy time series, differentiate between traditional and fuzzy time series. And forecast the fuzzy data using Mamdami's method.

\subsection{Title: Forecasting Enrollments using Fuzzy Time Series. Part I. Fuzzy Sets and Systems 54, 1-9}

Publisher: Mid-South Educational Research Association (20th, Lexington, KY, November 12-15, 1991)

Author: Song, Qiang, Chissom, Brad S

Theory: Conceptual introduction of fuzzy time series. Forecasted enrollment using a first-order time -invariant model and model was evaluated using the conventional linear regression technique then predicted values were compared with the actual enrollments and fuzzy time series results.

\section{Critical Review:}

Methodology Used: First order time-invariant model

Data Used: Alabama University enrollment data from 19711990.

Paper Conclusion: Compared the FTM (First order timeinvariant model) method predicted values with the non linear regression models. Founded that when the quadratic term was included then FTM had better result, but when the cubic term was included then non-linear regression model had better result. In future work, authors mentioned two different approaches using fuzzy time series. The first one was used the multi models and second one was used the feed-back prediction model.

Own Conclusion: This paper introduce the concept of fuzzy time series and presented a model to forecast the Alabama University enrollments data set using first order time invariant model. And then predicted results compared with the actual enrollment values and conventional linear regression values.

\subsection{Title: Forecasting enrollments with fuzzy time series - Part II}

Publisher: Article in Fuzzy Sets and Systems 62- Feb, 1994, North Holland

\begin{abstract}
Author: Qiang Song, Brad S. Chissom
Theory: This paper was a continuation of Forecasting Enrollments using Fuzzy Time Series. Part I. Fuzzy Sets and Systems 54, 1-9 $9^{[2]}$. In that, author applied first order time variant model to forecast the Alabama University enrollment data from 1971 to 1991 . The output of the proposed method interpreted by using three layer back-propagation neural network and then three different defuzzification methods were compared.
\end{abstract}

\section{Critical Review:}

Methodology Used: First order time-variant model

Data Used: Alabama University enrollment data from 19711992.

Paper Conclusion: Proposed a first order time variant model and forecast the enrollment. And defuzzify the output of the model by a trained three layer back propogation neural network. And then they had compared the three different defuzzification methods and resulted that the neural network provided best result.

Own Conclusion: This paper continued work of Forecasting Enrollments using Fuzzy Time Series. Part I. Fuzzy Sets and Systems 54, 1-9 $9^{[2]}$. In which the first order time variant model proposed and to check its feasibility forecasted the Alabama University enrollment data from 1971 to 1991 . Then the model output defuzzified with the three layer back propogation neural network. In last, they had presented the comparison among three different defuzzification methods and found that neural network yielded best result in terms of smallest average forecasting error.

\subsection{Title: A New Method to Forecast Enrollments using Fuzzy Time Series}

Publisher: International Journal of Applied Science and Engineering. 2004.2, 3:234-244

Author: Shyi-Ming Chen and Chia- Ching Hsu

Theory: Proposed a method belongs to the first order and time variant methods which used to forecast the enrollments based on fuzzy time series. Authors had taken the Alabama University historical enrollments data from 1972 to 1992. And the proposed method results had compared with different methods having same data set in terms of mean square error (MSE) rate. In comparison, found that proposed method had least MSE than other methods.

\section{Critical Review:}

Methodology Used: Proposed method belongs to the class of first order and time variant methods

Data Used: Alabama University enrollments data from 1972 to 1992

Paper Conclusion: Proposed a new method belongs to first order and time variant methods. Proposed method forecast the enrollment data based on fuzzy time series and the results were compared with different methods using same data. Conclude that the proposed method gave higher forecasting accuracy rate in term of least MSE than other methods. In their future work, they discussed to extend their proposed method with different forecasting problems which based on fuzzy time series and to proposed a new method for forecasting enrollments which based on fuzzy neural network to obtain a higher forecasting accuracy rate.

Own Conclusion: Proposed a method belongs to the first order and time variant methods used to forecast the enrollments based on fuzzy time series and the resulted outcomes were compared with different methods. Conclude that the proposed method provide higher accuracy rate in terms of least MSE rate.

\subsection{Title: Forecasting enrollments based on fuzzy time series}

Publisher: Fuzzy Sets and Systems 8961, 19 Elsevier Science B.V

\section{Author: Shyi-Ming Chen}

Theory: Proposed a new method to forecast university enrollments based on fuzzy time series. The proposed method compared with the Song Chissom (1993a) found to be more efficient. Proposed method used simplified arithmetic 
operations rather than complicated max-min operations. Robustness of proposed method was tested by using not accurate historical data in that case method gave better forecasted values.

\section{Critical Review:}

Methodology Used: Simplified method using arithmetic operations.

Data Used: Alabama University enrollments data from 1972 to 1992.

Paper Conclusion: Proposed method forecasted fuzzy time series enrollment data. Robustness of proposed method tested by forecasted non accurate data. Efficiency of proposed method depended on the usage of simplifies operations rather than complex max-min compositions.

Own Conclusion: This paper proposed method to forecast fuzzy time series data. In which used simplified operations rather than max-min compositions used in Sing Chissom (1993a). Robustness of proposed method tested by forecasted non accurate data.

\subsection{Title: Fuzzy Metric Approach for Fuzzy Time Series Forecasting based on Frequency Density Based Partioning}

Publisher: World Academy of Science, Engineering and Technology 34, 2007

Author: Tahseen Ahmed Jilani, Syed Muhammad Aqil Burney, and Cemal Ardil

Theory: Proposed method used historical enrollment data for forecasting based on frequency density partitioning. Proposed method related to the class of $\mathrm{k}^{\text {th }}$ order and time variant methods. Proposed method had resulted with smallest forecasted values in terms of AFER (Average Forecasting Error Rate) and MSE (Mean Square Error) by comparing with different method using same data set.

\section{Critical Review:}

Methodology Used: Forecasted enrollment based on frequency density partitioning.

Data Used: Alabama University Enrollment data from 1971 to 1992

Paper Conclusion: Proposed method had resulted with smallest forecasted values in terms of AFER (Average Forecasting Error Rate) and MSE (Mean Square Error) by comparing with different method using same data set. In their work, they mentioned to proposed forecasted method based on semi-parametric and fuzzy parametric approaches for better smallest values.

Own Conclusion: Proposed method forecasted enrollment data based on frequency density portioning. Proposed method belongs to the kth order and time variant methods. Forecasted model return result in terms of smallest values of AFER and MSE. In future they discussed about to forecast model based on semi-parametric and fuzzy parametric approaches.

\subsection{Title: Multivariant High Order Fuzzy Time Series Forecasting for Car Road Accidents}

Publisher: International Journal of Computational Intelligence 4; 12008
Author: Tahseen A.Jilani, S.M.Aquil Burney, and C. Ardil

Theory: In this paper, proposed a new multivariate fuzzy time series forecasting method for forecasting Belgium car accidents data and calculated actuaries in pattern analysis of causalities in car road accidents.

In fuzzy time series, actuaries were referred to the fuzzy premium and fuzzy underwriting of car insurance and life insurance for car insurance. By using the risk classification provided by National Institute of Statistics of Belgium for each road, method had forecasted the AFER (Average Forecasting Error Rate) values to predict underwriting and premium rate of insurance policy holders.

\section{Critical Review:}

Methodology Used: Modified method based on m-factors of high order fuzzy time series

Data Used: National Institute of Statistics, Belgium car accidents data from 1974 to 2005

Paper Conclusion: In this paper extended the work of Lee ${ }^{[25]}$. Proposed modified model based on $\mathrm{m}$-factors of high order fuzzy time series. A multivariate fuzzy time series forecasted model used the National institute of Statistics, Belgium car accidents data from 1974 to 2005 which focused on yearly road accident causalities, mortally wounded, died. In a month, severely wounded and light causalities.

Own Conclusion: A multivariate fuzzy model proposed, based on $\mathrm{m}$-factors of high order fuzzy time series. Proposed model used the Belgium car accidents data from 1974 - 2005 for forecasting values in terms of AFER. And comparison was done with different previous methods that using same data set.

\subsection{Title: Fuzzy metric approach for fuzzy time series forecasting based on frequency density based portioning}

Publisher: World Academy of Science, Control and Information Engineering, Vol: 4, No: 7, 2010

Author: Tahseen Ahmed, Syed, and Ardil

Theory: Proposed a fuzzy time series method based on frequency density based partitioning for forecasting enrollment. The method was belongs to the class of kth order and time-variant method. The results of proposed method had compared with pre-existing methods in terms of AFER and MSE rate and provide the smallest error rates.

\section{Critical Review:}

Methodology Used: Fuzzy metric approach based on frequency density based portioning.

Data Used: Alabama University Enrollment data from 1972 to 1992

Paper Conclusion: Proposed a frequency density based partitioning method, applied improved fuzzy metric for forecasting. The method belonged to class of kth order and time-variant method. Calculated results in terms of AFER and MSE compared with different methods and founded the smallest error rates.

Own Conclusion: Proposed a fuzzy time series method proposed, based on frequency density based partitioning for forecasting enrollment. Results were calculated in terms of AFER and MSE values, and founded that proposed method having smallest error rate while comparing with different 
method using same data set.

\subsection{Title: A Multivariate Heuristic Model for Fuzzy Time Series Forecasting}

Publisher: IEEE TRANSACTIONS ON SYSTEMS, MAN, AND CYBERNETICS-PART B: CYBERNETICS, VOL. 37, and NO. 4, AUGUST 2007

Author: Kun-Huang Huarng, Tiffany Hui-Kuang Yu, and Yu Wei Hsu

Theory: Proposed a multivariate heuristic function model integrated with univariate fuzzy time series model. Various variables were applied in heuristic function for forecast the Taiwan Stock Index. Then fuzzy relationship procedure applied and selected proper fuzzy set. And the determined results were compared with the matrix computation and found better result.

\section{Critical Review:}

Methodology Used: Multivariate Heuristic Model integrated with Univariate Model

\section{Data Used: Taiwan Stock Index for 2001}

Paper Conclusion: Proposed a multivariate heuristic function model integrated with univariate fuzzy time series model. Various variables were applied in heuristic function for forecast the Taiwan Stock Index. Then fuzzy relationship procedure applied and selected proper fuzzy set. And the determined results were compared with the matrix computation and found better result.

Own Conclusion: Proposed multivariate heuristic function model, integrate with univariate fuzzy time series model. Various variables were applied in heuristic function for forecast the Taiwan Stock Index. Then fuzzy relationship procedure applied and selected proper fuzzy set. And the determined results were compared with the matrix computation and found better result.

\subsection{Title: Forecasting Enrollment Model}

Based on First-Order Fuzzy Time Series

Publisher: Proceedings of world academy of science, Engineering and technology volume 1 January 2005 ISSN $1307-6884$

\section{Author: Melike, Konstant}

Theory: Proposed a modified time-invariant fuzzy time series method for forecasting the Alabama university enrolment data. Unlike Song-Chissom and Chen approaches, the proposed method used the variations of data rather than utilization of actual enrollment data. The fuzzy relationship had been calculated for the forecasting. For examine the method, the average forecasting error rate was calculated and compared with different models using same dataset.

\section{Critical Review:}

Methodology Used: Time-invariant fuzzy time series

Data Used: Alabama university enrollment data from 1971 to 1992

Paper Conclusion: A time-invariant fuzzy time series model presented using the university of Alabama enrollment data. The proposed method basically used the variations available in enrollment data. Then, the results were compared in terms of average forecasting error rate with different methods having same dataset.
Own Conclusion: Proposed modified time-invariant fuzzy time series method for forecasting the Alabama university enrolment data (1971 to 1992). The proposed method used the variations available in enrollment data rather than actual enrollments. And the results were compared with the different methods that are using the same dataset in terms of average forecasting error rate.

\subsection{Title: A Hybrid Algorithm to Forecast Enrollment Based on Genetic Algorithms and Fuzzy Time Series}

Publisher: The International Arab Journal of Information Technology, Vol. 11, No. 3, May 2014

Author: Hanseen, Kais, Abdul-Ghafoor

Theory: Proposed hybrid algorithm for forecasting Alabama university enrollment data based on fuzzy time series and genetic algorithm. Firstly, determined the best interval having least fittest function value and then secondly, applied the fuzzy forecasting procedure. Proposed method results were calculated in terms of MSE (mean square error rate) compared with different pre-existing methods and founded that method having least error rate.

\section{Critical Review:}

Methodology Used: Hybrid method based on fuzzy time series and genetic algorithms

Data Used: Alabama University Enrollment data from 1948 to 2010

Paper Conclusion: Proposed a hybrid algorithm based on fuzzy time series and genetic algorithm. Result calculated in MSE and compared the method with different methods using same enrollment data. As a result, proposed method had least value of MSE.

Own Conclusion: Proposed hybrid algorithm that based on fuzzy time series and genetic algorithms, for forecasting Alabama university enrollment data. Proposed method had least MSE value as compared with pre-existing methods.

\subsection{Title: A Model-Based Approach to the Analysis of Patterns of Length of Stay in Institutional Long-Term Care} Publisher: IEEE TRANSACTIONS ON INFORMATION TECHNOLOGY IN BIOMEDICINE, VOL. 10, NO. 3, JULY 2006

Author: Haifeng, Thierry, and Peter $\mathrm{H}$

Theory: Proposed a modular approach for analyzing and differences in LOS (Length of Stay) patterns of public funded residents in Institutional LTC (Long Term Care) routinely administrative data provided by London Borough of Merton.

\section{Critical Review:}

Methodology Used: A Model-Based Approach to the Analysis of Patterns of Length of Stay

Data Used: London Borough of Merton administrated dataset collected by routinely

Paper Conclusion: Proposed a modular approach for analyzing and mining an administration social-care data set. Model had analyzed high level behavior of Institutional LTC data and provided the important information regarding how to planned and managed the system. 
Own Conclusion: Proposed a modular approach for analyzing and mining the pattern of public funded residents in Institutional LTC administrative data provided by London Borough of Merton. And Model had provided the important information regarding planning and managing the system.

\subsection{Title: A New Method to Forecast Enrollments using Fuzzy Time Series and Clustering Techniques}

Publisher: Proceedings of the Eighth International Conference on Machine Learning and Cybernetics, Baoding, 12-15 July 2009

Author: Kurniawan, Shyi-Ming Chen

Theory: Proposed a method based on fuzzy time series and automatic clustering algorithm. Method had used the first order and high order fuzzy time series, respectively. And the forecasted model results were calculated in terms of mean square error and proposed method compared with pre-existing methods found the smallest value of MSE.

\section{Critical Review:}

Methodology Used: Fuzzy Time Series (first order and high order, respectively) and automated clustering algorithm.

Data Used: Alabama University Enrolment data from 1971 to 1992

Paper Conclusion: Proposed a method based on fuzzy time series and automatic clustering algorithm. And the forecasted results in terms of mean square error and compared with other methods having same data set and founded the least MSE value.

Own Conclusion: Presented a method based on fuzzy time series and automatic clustering algorithm. Model used Alabama enrollment data. The method had used the first order fuzzy time series and high order fuzzy time series, respectively. And the forecasted results in terms of mean square error and compared with other methods having same data set and founded the least MSE value.

\subsection{Title: A Novel Approach of Genetic Algorithm in prediction of Time Series Data}

Publisher: International Journal of Computer Applications on Advanced Computing and Communication Technologies for HPC Applications - ACCTHPCA, June 2012

Author: Satyendra Nath Mandal, Arghya Gosh, Subhojit Roy, J.Pal and S.R Bhadra

Theory: The genetic algorithm proposed for forecasting time series data. A comparison had made with different pre-existing methods like statistical, artificial neural network and fuzzy system and found better result.

\section{Critical Review:}

Methodology Used: Genetic Algorithm used

Data Used: Data Set had been collected from research paper Satyendra Nath Mandal ${ }^{[26]}$. The data related to the mustard plant shoot length.

Paper Conclusion: The genetic algorithm proposed for forecasting time series data. A comparison had made with different pre-existing methods like statistical, artificial neural network and fuzzy system and found better result.
Own Conclusion: The genetic algorithm proposed, model forecast fuzzy time series data contain the shoot length data of mustard plant. A comparison had made with different preexisting methods like statistical, artificial neural network and fuzzy system and found better result.

\subsection{Title: A Survey on Data Mining Approaches for Healthcare}

Publisher: International Journal of Bio-Science and BioTechnology, 2013

Author: Divya Tomar, Sonali Agarwal

Theory: Paper presented a survey on data mining and its approaches, firstly explained the data mining and its importance in healthcare domain and then hunts different data Mining Techniques like clustering, classification, association and regression in Healthcare domain. Also had mentioned its advantages and disadvantages, also included its applications, challenges and future issues of data mining in healthcare field.

Critical Review:

Methodology Used: Survey of Data Mining

Data Used: none

Paper Conclusion: This paper presented a survey on Data Mining and its approaches, and hunted different data mining techniques in healthcare domain. Also had mentioned its advantages and disadvantages, also included its applications, challenges and future issues of data mining in healthcare field.

Own Conclusion: Paper present survey on data mining and its approaches, firstly had explained the data mining and its importance in healthcare domain then hunts different data Mining Techniques like clustering, classification, association and regression in Healthcare domain. Also had mentioned its advantages and disadvantages, also included its applications, challenges and future issues of data mining in healthcare field.

\subsection{Title: Adaptive Time-Variant Models for Fuzzy-Time-Series Forecasting} Publisher: IEEE TRANSACTIONS ON SYSTEMS, MAN, AND CYBERNETICS-PART B: CYBERNETICS, VOL. 40, and NO. 6, DECEMBER 2010

Author: Wai-Keung Wong, Enjian Bai, and Alice Wai-Ching Chu

Theory: Proposed an adaptive time-variant fuzzy-time-series forecasting model (ATVF) for forecasting enrollment, temperature, stock indices and other domain. The proposed method automatically adapted the window size of fuzzy time series on basis of prediction accuracy in training phase and utilized heuristic rules for producing the forecasted values in testing phase.

\section{Critical Review:}

Methodology Used: Adaptive time-variant fuzzy-time series forecasting method

Data Used: Alabama University Enrollment data and Taiwan Stock Exchange Capitalization Weighted Stock Index (TAIEX)

Paper Conclusion: Proposed an adaptive time-variant fuzzytime series forecasting model (ATVF) for forecasting enrollment, temperature, stock indices and other domain. The forecasted method efficiency had compared with simulated and real time-series data. In future work, c-means clustering 
or genetic algorithm used to improvised the model or integrated the model.

Own Conclusion: An adaptive time-variant fuzzy-time-series forecasting model proposed, provide some heuristic values in training phase and then utilized the values in testing phase

\subsection{Title: Forecasting enrollment with high-order fuzzy time series}

Publisher: IEEE 2000

\section{Author: Chao-Chin Tsai, Shun-Jyh Wu}

Theory: Proposed the high-order forecasting model integrated with the first-order fuzzy time series forecasting models. Proposed method had generated results in terms of root mean square error and concluded with improvised results while comparing with Song-Chissom method. ${ }^{[1][2]}$

\section{Critical Review:}

Methodology Used: High order forecasting method integrated with first order fuzzy time series forecasting models

Data Used: Alabama University Enrollment data from 1971 to 1992

Paper Conclusion: Proposed high-order forecasting model integrated with first order fuzzy time series models. Provide improvised results in terms of root mean square error.

Own Conclusion: Proposed the high-order forecasting model, integrated with the first-order fuzzy time series forecasting models. Proposed method had generated results in terms of root mean square error and concluded with improvised results while comparing with Song-Chissom method. ${ }^{[1][2]}$

\subsection{Title: Soft Computing Model to Predict Average Length of Stay of Patient}

Publisher: Springer-Verlag Berlin Heidelberg 2011

Author: Bindu Garg, M.M. Sufyan Beg, A.Q. Ansari, and B.M. Imran

Theory: Proposed a dynamic computational method based on fuzzy time series, using soft computing techniques for forecasting patient average length of stay. For the algorithm implementation, used event discretization function, frequency density based partitioning procedure and optimization of fuzzy relationship procedure. The forecasted results had calculated and compared with pre-existing methods in terms of AFER (average forecasting error rate) and MSE (mean square error), determined the lower value that means higher forecasting error rate.

\section{Critical Review:}

Methodology Used: A dynamic computational model using soft computing techniques

Data Used: Alabama University historical data of year 2008 and 2009

Paper Conclusion: Proposed a dynamic computational method based on fuzzy time series, using soft computing techniques for predicting and forecasting average length of stay of patient. Results were calculated in terms of AFER and MSE and found better forecast values when compared with other methods having same data set. In future work, proposed model can be optimized with genetic algorithm.
Own Conclusion: Proposed a dynamic computational method, based on fuzzy time series, using soft computing techniques for forecasting patient average length of stay. Results were calculated in terms of AFER and MSE and found better forecasted values when compared with other methods having same data set. In future work, proposed model can be optimized with genetic algorithm.

\subsection{Title: Fuzzy Time Series Prediction Model}

Publisher: Springer-Verlag Berlin Heidelberg 2011

Author: Bindu Garg, M.M. Sufyan Beg, A.Q. Ansari, and B.M. Imran

Theory: Proposed soft computing time series model, implemented event discretization function, frequency density based partitioning procedure and optimization of fuzzy relationship procedure. Proposed method using Alabama university enrollment data and results calculated in terms of AFER and MSE and found better forecasted values when compared with other methods using same data set.

Critical Review:

Methodology Used: Soft computing time series model

Data Used: Alabama University enrollment data from 1971 to 1992

Paper Conclusion: Proposed a soft computing time series model based on fuzzy time series for predicting and forecasting average length of stay of patient. Results were calculated in terms of AFER and MSE and found better forecasted values when compared with other methods having same data set. In future work, proposed model can be optimized with genetic algorithm.

Own Conclusion: Proposed soft computing time series model based on fuzzy time series for forecasting patient average length of stay. Results were calculated in terms of AFER and MSE and found better forecasted values when compared with other methods having same data set. In future work, proposed model can be optimized with genetic algorithm.

\subsection{Title: On Fuzzy Time Series Method}

Publisher: $20103^{\text {rd }}$ International Symposium on Knowledge Acquisition and Modeling

Author: Yu Yan-Hua and Song

Theory: Paper presented to analyze the properties and methods of fuzzy time series. And had explained theory structure, forecasting procedure and its calculations, and discussed about the applications of fuzzy time series which had characterized through comparison between traditional and fuzzy time series.

\section{Critical Review:}

Methodology Used: Fuzzy time series model

Data Used: Alabama University enrollment data from 1971 to 1992

Paper Conclusion: Proposed a fuzzy time series forecasting model to analyze the methods and properties of fuzzy time series. And the proposed method had compared with different methods.

Own Conclusion: Proposed fuzzy time series forecasting model to analyze the properties and methods of fuzzy time series. And proposed method had compared with different 
existing methods having same data set.

\subsection{Title: Predicting Emergency Department Length of Stay Using Quantile Regression}

Publisher: IEEE2009

Author: Ru Ding, Jennifer Lee, Jeffrey, Scott L. and Dominik

Theory: Proposed a multivariant quantile regression model in three phases of ED care functions as day, time, age and gender, mode of arrival, insurance status, acquity level, and chief complaint. In this paper used academic ED data from a tertiary-care medical centre.

\section{Critical Review:}

Methodology Used: Multivariant quantile regression model

Data Used: Academic ED data of tertiary-care medical centre from October 1, 2005 to September 30, 2006

Paper Conclusion: A multivariant quantile regression model presented in three phases of ED care functions for that used the academic ED data. Concluded, that ED varied significantly among patients in a forecasted manner which was explained by information at triage.

Own Conclusion: Proposed multivariant quantile regression model in three phases of ED care functions as day, time, age and gender, mode of arrival, insurance status, acquity level, and chief complaint. In this paper used academic ED data from a tertiary-care medical centre. Concluded, that ED varied significantly among patients in a forecasted manner which was explained by information at triage.

\subsection{Title: Learning Predictive Models from Integrated Healthcare Data: Extending Pattern-based and Generative Models to Capture Temporal and Cross-Attribute Dependencies}

Publisher: Supported by FCT under research project

Author: Rui Henriques and Antunes

Theory: Proposed predictive model into two parts, firstly data mining had combined with multiple time sequence and secondly proposed two predictive models one deterministic (P2MID) and one generative (M2ID). And proposed model was compared with other models.

\section{Critical Review:}

Methodology Used: Proposed a Predictive model used data mining, deterministic (P2MID) and generative (M2ID)

Data Used: healthcare heritage prize database ${ }^{[27]}$

Paper Conclusion: Proposed learning predictive model that support medical decisions using healthcare data. Model had integrated with two things that data mining and P2MID and M2ID methods.

Own Conclusion: Proposed the predictive model into two parts firstly data mining had combined with multiple time sequence and secondly proposed two predictive models one deterministic (P2MID) and one generative (M2ID). And proposed model was compared with other models.

\subsection{Title: Patient grouping optimization using a hybrid Self-Organizing Map and Gaussian Mixture Model for length of stay-based clustering system \\ Publisher: IEEE, 2010}

Author: Florin, Elia, Smaranda and Marina

Theory: Paper proposed two approaches one is Selforganizing Map (SOM) and other is Gaussian Mixture Model (GMM). SOM used to train the data set whereas GMM used to calculate the result. At last, proposed method results were calculated with other methods found better results.

\section{Critical Review}

Methodology Used: Self-Organizing Map and Gaussian Mixture Model

Data Used: Surgical Australia Adelaide tertiary hospital dataset from 4 February, 1997 to 30, June 1998

Paper Conclusion: Proposed method with self-organizing map and Gaussian mixture model for clustering and determining results. Then result had calculated and compared with other methods.

Own Conclusion: This paper proposed Self-organizing Map (SOM) and Gaussian Mixture Model (GMM). SOM used to train the data set whereas GMM used to calculate the result. At last, proposed method results were calculated with other methods found better results.

\subsection{Title: Using Survival Models to Analyze the Effects of Social Attributes on Length of Stay of Stroke Patients} Publisher: IEEE, 2009

Author: Chee-Keong Kwoh, Kheng-Hock Lee, and Truc-Viet Le

Theory: Proposed Survival Method to analyze the effects of Social Attributes on Length of Stay of patient by using Stroke Patient data collected from Singapore General Hospital (SGH) for $2004-2007$. Paper described the significance of social attributes on healthcare and effects on utilization of healthcare resources.

\section{Critical Review:}

Methodology Used: Survival analysis method

Data Used: Singapore General Hospital (SGH) Stroke Patient data $2004-2007$

Paper Conclusion: Paper had discussed survival analysis of stroke patient data to calculate patient length of stay. Paper described the significance of social attributes on healthcare and effects on utilization of healthcare resources.

Own Conclusion: Proposed Survival method to analyze the effects of Social Attributes on Length of Stay of patient by using Stroke Patient data collected from Singapore General Hospital (SGH) for 2004 - 2007. Paper described the significance of social attributes on healthcare and effects on utilization of healthcare resources.

\section{DISCUSSION OVER REVIEWED ALGORITHMS}

In this section, we have discussing about the pre-existing work and its implementations in reference of patient length of 
stay. Song, Chissom ${ }^{[1,2 \text {, and } 3]}$ proposed time-invariant method to forecast time series enrollment data. Shyi- ming Chen ${ }^{[4,5]}$ proposed a method belong to first-order fuzzy time series and time invariant to forecast enrollment data. Tahseen and Ardil $[6,7$, and 8$]$ proposed fuzzy metric approach to forecast enrollment data. Huang ${ }^{[9]}$ proposed multivariant heuristic model to forecast enrollment fuzzy time series data. Melike [10] proposed first order time series model to forecast enrollment data set. Hanseen ${ }^{[11]}$ proposed hybrid algorithm using genetic algorithm and fuzzy time series. Haifeng ${ }^{[12]}$ proposed model based approach for patient length of stay pattern analysis. Kurniawan ${ }^{[13]}$ proposed method using fuzzy time series and clustering techniques for forecasting enrollments. Satyendra Nath ${ }^{[14]}$ proposed genetic method approach for time series forecasting. Wai-keung ${ }^{[16]}$ proposed adaptive time-variant model for forecasting fuzzy time series data. Chao-Ching Tsai ${ }^{[17]}$ proposed high-order fuzzy time series model. Bindu Garg ${ }^{\text {[18. }}{ }^{19]}$ proposed soft computing model using fuzzy time series to forecast patient length of stay interms of AFER and MSE. Yu-Yang -Hua ${ }^{[20]}$ proposed model using fuzzy time series to forecast enrollment data.

Forecasting prediction play a significant role inpatient healthcare plans and medical insurance plans. Some applications are built in that reference but these applications are complex in healthcare system which is impractical to implementation in real life. So, it's important to develop scientifically robust and practical existent model for forecasting patient length of stay.

\section{CONCLUSION}

Forecasting In-patient stay is our prime concern for patient diagnosis of their diseases. So, it's important for hospitals to efficiently plan and manage services and resources for better healthcare. In this paper, presents a review of related work and their proposed method implementation. Bindu Garg ${ }^{[18,19]}$ proposed soft computing model using fuzzy time series and forecast fuzzy data in terms of AFER value is $0.34 \%$ and MSE value is 9917.17. Thereafter, different research paper analyzed and prepared a critical review that presented in section 3 and 4.

\section{FUTURE WORK}

Forecasting prediction play a significant role inpatient healthcare plans and medical insurance plans. Some applications are built in that reference but these applications are complex in healthcare system which is impractical to implementation in real life. So, it's important to develop scientifically robust and practical existent model for forecasting patient length of stay. Proposed new model will optimize with genetic algorithm which achieved better results in terms of AFER and MSE. Develop a healthcare system model for hospitals administrations and patients which efficiently plan and manage services.

\section{REFERENCES}

[1] Q. Song, B.S Chissom, An article in Fuzzy Sets and Systems March (1993) 267-277 Elsevier Science Publisher B.V. A Fuzzy Time Series and its model.

[2] Song, Qiang, Chissom and Brad S., Mid-South Educational Research Association 20th, Lexington, KY, November 12-15, 1991. Forecasting Enrollments using Fuzzy Time Series. Part I. Fuzzy Sets and Systems 54, $1-9$.

[3] Q. Song, B. S Chissom, Article in Fuzzy Sets and Systems 62- Feb, 1994, North Holland. Forecasting enrollments with fuzzy time series - Part II.

[4] Shyi-Ming Chen and Chia- Ching Hsu, International Journal of Applied Science and Engineering. 2004.2, 3:234-244. A New Method to Forecast Enrollments using Fuzzy Time Series.

[5] Shyi-Ming Chen, Fuzzy Sets and Systems 8961, 19 Elsevier Science B.V. Forecasting enrollments based on fuzzy time series.

[6] Tahseen Ahmed Jilani, Syed Muhammad Aqil Burney, and Cemal Ardil, World Academy of Science, Engineering and Technology 34, 2007. Fuzzy Metric Approach for Fuzzy Time Series Forecasting based on Frequency Density Based Partioning.

[7] Tahseen A.Jilani, S.M.Aquil Burney, and C. Ardil, International Journal of Computational Intelligence 4;1 2008. Multivariant High Order Fuzzy Time Series Forecasting for Car Road Accidents.

[8] Tahseen Ahmed, Syed, and Ardil, World Academy of Science, Control and Information Engineering, Vol:4, No: 7, 2010, Fuzzy metric approach for fuzzy time series forecasting based on frequency density based portioning.

[9] Kun-Huang Huarng, Tiffany Hui-Kuang Yu, and Yu Wei Hsu, IEEE TRANSACTIONS ON SYSTEMS, MAN, AND CYBERNETICS-PART B: CYBERNETICS, VOL. 37, NO. 4, AUGUST 2007. A Multivariate Heuristic Model for Fuzzy Time Series Forecasting.

[10] Melike, Konstant, Proceedings of world academy of science, Engineering and technology volume 1 January 2005 ISSN 1307-6884. Forecasting Enrollment Model Based on First-Order Fuzzy Time Series.

[11] Hanseen, Kais, Abdul-Ghafoor, The International Arab Journal of Information Technology, Vol. 11, No. 3, May 2014. A Hybrid Algorithm to Forecast Enrollment Based on Genetic Algorithms and Fuzzy Time Series.

[12] Haifeng, Thierry, and Peter H, IEEE TRANSACTIONS ON INFORMATION TECHNOLOGY IN BIOMEDICINE, VOL. 10, NO. 3, JULY 2006. A Model-Based Approach to the Analysis of Patterns of Length of Stay in Institutional Long-Term Care.

[13] Kurniawan, Shyi-Ming Chen, Proceedings of the Eighth International Conference on Machine Learning and Cybernetics, Baoding, 12-15 July 2009. A New Method to Forecast Enrollments using Fuzzy Time Series and Clustering Techniques.

[14] Satyendra Nath Mandal, Arghya Gosh, Subhojit Roy, J.Pal and S.R Bhadra, International Journal of Computer Applications on Advanced Computing and Communication Technologies for HPC Applications ACCTHPCA, June 2012. A Novel Approach of Genetic Algorithm in prediction of Time Series Data.

[15] Divya Tomar, Sonali Agarwal, International Journal of Bio-Science and Bio-Technology, 2013. A Survey on Data Mining Approaches for Healthcare.

[16] Wai-Keung Wong, Enjian Bai, and Alice Wai-Ching Chu, IEEE TRANSACTIONS ON SYSTEMS, MAN, AND CYBERNETICS-PART B: CYBERNETICS, VOL. 40, NO. 6, DECEMBER 2010. Adaptive TimeVariant Models for Fuzzy-Time-Series Forecasting. 
[17] Chao-Chin Tsai, Shun-Jyh Wu, IEEE 2000. Forecasting Enrollment with High-order Fuzzy Time Series.

[18] Bindu Garg, M.M. Sufyan Beg, A.Q. Ansari, and B.M. Imran, Springer-Verlag Berlin Heidelberg 2011. Soft Computing Model to Predict Average Length of Stay of Patient.
[19] Bindu Garg, M.M. Sufyan Beg, A.Q. Ansari, and B.M. Imran, Springer-Verlag Berlin Heidelberg 2011. Fuzzy Time Series Prediction Model.

[20] Yu Yan-Hua and Song, $20103^{\text {rd }}$ International Symposium on Knowledge Acquisition and Modeling. On Fuzzy Time Series Method. 